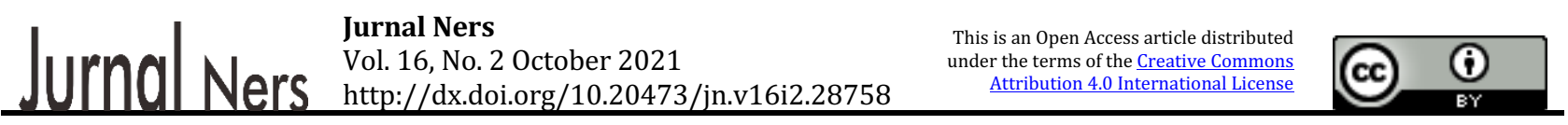

Original Research

\title{
The Effectiveness of a Nurse-led Team-Based Hypertension Management among People with Uncontrolled Hypertension in a Community Hospital, Thailand
}

\author{
Nongnut Oba ${ }^{1}$ and Navarat Chutipanyaporn ${ }^{2}$ \\ ${ }^{1}$ Faculty of Nursing, Naresuan University, Phitsanulok Province, Thailand \\ 2 Bang Rakam Hospital, Bang Rakam District, Phitsanulok Province, Thailand
}

\begin{abstract}
Introduction: Uncontrolled blood pressure of people with hypertension remains a major public health issue. The purpose of this research was to evaluate the effectiveness of a Nurse-led Team-based Hypertension Management Program (NTHMP) among people with uncontrolled hypertension.
\end{abstract}

Methods: This quasi-experimental one-group pre-posttest design research was done to evaluate the effectiveness of a NTHMP in a community hospital in Thailand. The sample was thirty people with uncontrolled hypertension who received outpatient care in a community hospital in Thailand. They participated in three months NTHMP which included 1) team-approached health education, 2) medication administration support, 3) motivation interviewing on behavioral adjustment and 4) home blood pressure monitoring for three months. Outcomes of the program; systolic blood pressure, diastolic blood pressure, hospital admissions with signs of hypertensive urgency were analyzed by using frequency, percentage, mean, standard deviation, and repeated measured ANOVA.

Results: The results indicated that people with uncontrolled HT had lower systolic blood pressures and diastolic blood pressure compared with baseline levels ( $\mathrm{p}<0.001)$, and no hospital admissions.

Conclusion: This program provided evidence for nurses to manage blood pressure control in people with hypertension within a collaboration with multidisciplinary team members in the community hospital.

\section{ARTICLE HISTORY}

Received: July 30, 2021

Accepted: November 29, 2021

\section{KEYWORDS}

covid-19; online clinical learning; nursing student

\section{CONTACT}

Nongnut Oba

$\triangle$ nongnut@nu.ac.th

$\equiv$ Faculty of Nursing, Naresuan University, Phitsanulok Province, Thailand

Cite this as: Oba, N., \& Chutipanyaporn, N. (2021). The Effectiveness of a Nurse-led Team-Based Hypertension Management among People with Uncontrolled Hypertension in a Community Hospital, Thailand. Jurnal Ners, 16(2). 169-176. doi:http://dx.doi.org/10.20473/jn.v16i2.28758

\section{INTRODUCTION}

Hypertension (HT) exerts a staggering worldwide burden on human quality of life and healthcare system resources via contribution to increased mortality and risk of cardiovascular diseases, chronic kidney disease and stroke globally (World Health Organization, 2019). Worldwide, raised blood pressure (BP) is estimated to cause 7.5 million deaths, about $12.8 \%$ of the total of all deaths (Basile \& Bloch, 2019). In general, lifestyle management is recommended for people with mild HT (average BP = 140-159/90- 99), low-risk for cardiovascular diseases and no co-morbidities (Whelton et al., 2018). Antihypertensive drug with lifestyle management was used for lowering BP in the moderate HT (Flack
\& Adekola, 2019; Thai Hypertension Society, 2019). The new ACC/AHA hypertension guidelines indicated the goal of hypertensive care is that people with HT have an SBP of $130 \mathrm{mmHg}$ or lower and DBP of 80 mmHg or lower (Flack et al., 2019). However, the Thai Hypertension Society indicated the patient's BP should initially be lowered to under 140/90 mmHg and if the patient shows good tolerance to treatment then it should be further treated to $<130 / 80 \mathrm{mmHg}$ (Thai Hypertension Society, 2019). Therefore, people with HT who can manage their medication taking and behavioral life style well until they achieve a BP < $140 / 90 \mathrm{mmHg}$ are defined as having controlled HT, while others having a BP $>140 / 90 \mathrm{mmHg}$ two times continuous have uncontrolled HT. 
On the Health Disease Control (HDC) dashboard of the Ministry of Public Health (MOPH), data during 2017-2019, it was found that the prevalence rate of controlled HT was $35-50 \%$ in a cumulative data of district, provincial, and national levels (Health Data Center, 2020). These data affected Bang Rakam Hospital, a small community hospital in Phitsanulok province, Service area 2, MOPH which rethought and made a new plan to improve quality of hypertensive care.

Unhealthy lifestyle choices and non-adherence to medication are major causes of uncontrolled hypertension and are a high risk to progress to cardiovascular disease, chronic kidney disease and stroke that could threaten health and endanger life (Williams et al., 2018). Acute signs of very high blood pressure, which is called "hypertensive urgencies" have signs of stroke that threaten sudden death (Bakris, 2019). Practicing a healthy lifestyle; physical activity, healthy diet, restricted alcohol consumption, salt restriction, avoidance of tobacco use, and stress reduction and adherence to anti-hypertension medication are recommended in hypertension care (Alsaigh et al., 2017; Flack et al., 2019; Whelton et al, 2018).

Promoting lifestyle behavior changes among people with HT aimed at lowering BP to normal level is herculean. In the review, it was found that health education and practice on the DASH program (Seangpraw et al., 2019), aerobic exercise training (Bersaoui et al., 2020), supervised walking (Mandini et al., 2018), mindfulness (Ponte Márquez et al., 2019), small group discussion and feedback about hypertension (Thongdang \& Promsiripaiboon, 2015), and self-care management program (Plaiyod, Panpakdee, \& Taikerd, 2012) are helpful for lowering blood pressure among people with hypertension. Self-monitoring blood pressure (SMBP) significantly reduced office blood pressure and improved medication adherence (Muhammad, Jamial, \& Ishak, 2019). SMBP at home may increase patient engagement and improve BP control (Ho, Carnagarin, Matthews, \& Schlaich, 2018). SMBP and cointerventions (including systematic medication titration, health education, or lifestyle counselling) leads to clinically significant BP reduction (Tucker et al., 2017). A technique proved to have benefits to promote appropriate behaviors and decrease blood pressure levels among HT is motivational interviewing (MI). It was found that $\mathrm{MI}$ is powerful in reduction of SBP or DBP and weight reduction (Ozpulat \& Emiroglu, 2017), improves the adherence to a low-sodium diet, adherence to self-care behaviors, regular use of antihypertensive medications, increases or maintains physical activity (Silveira et al., 2019), evaluation of changes in daytime sleepiness, and cessation of smoking and cessation of drinking (Dechkong, 2017). It elicited behavioral changes by helping clients to explore and resolve ambivalence (Rollnick \& Allison, 2004).

At the primary care level in district hospitals, the healthcare team of hypertension management includes a physician, pharmacist, physical therapist and professional nurse. At this setting, a nurse who trained to be nurse practitioner (NP) usually acts as case manager, whereas the physician focuses on prescribing medication (Proia et al., 2014). Although hypertensive guidelines are recommended, a paradigm shift from fragmented care to a team approach is aimed at improving the quality of hypertensive care management (Flack et al., 2019; Thai Hypertension Society, 2019). Team-based care was able to increase the proportion of people with controlled BP and reduced both SBP and DBP, especially when pharmacists and nurses were part of the team (American College of Cardiology/American Heart Association Task Force, 2017). Team approach could be kept viable on hypertension management, the organizational leader must be committed and willing to allocate the necessary resources (World Health Organization, 2019). People with HT should be active partners, working with the health team to create a self-individualized plan (Patel et al., 2016).

Therefore, this research integrates concepts of hypertension management and team-based care to develop a Nurse-led Team-based Hypertension Management Program (NTHMP) aimed at improving blood pressure among people with uncontrolled HT.

\section{MATERIALS AND METHODS}

A one-group quasi-experimental repeated measure design was used as protocol. The program's effectiveness was tested by comparing the differences in SBP and DBP levels at before, the first and second month after the programmed interventions, and three months post-intervention of the sample. The number of hypertensive urgency and hospital admission by the sample post intervention was counted to calculate the effectiveness of the developed program.

The population was uncontrolled HT of chronic care clinic in Bang Rakam Hospital, a community hospital in Phitsanulok province, Service area 2, Ministry of Public Health, Thailand. They were 598 patients who met the inclusion criteria; 1) had $\mathrm{BP}>140 / 90 \mathrm{mmHg}$ two times and over 2) received hypertensive medication, and 3) able to participate in the program. If they had HT complication such as stroke, chronic kidney disease, and heart disease, they were excluded. If they changed hypertensive medication and/or moved to receive another health serviced and/or could not participate in all activities of the program, they were withdrawn. The sample size calculation of Polit and Beck using power analysis was employed to reduce the risk of type I error. The minimum level of significance $(\alpha)$ to estimate the number of sample size was 0.05 with the power of $0.80(1-\beta)$, which would yield a sample size of $n=30$. They were 598 patients who met the inclusion criteria, the investigator used simple random sampling for sample selection. The researcher listed all of the population members initially, and then each member was marked from 1 to 598. Random Number 
Generator Software, an internet application, was used for simple random sampling. The researcher followed the direction of generator software until receiving 30 selected numbers. The selected numbers were compared to an organized list for getting name and phone numbers of the sample. The professional nurse of the chronic care clinic who acted as coordinator, invited the selected sample to participate in the research project by phone call. If any sample was not willing to participate in the project, the researchers could select a new number by generator software.

There were two types of instruments used in this research: an intervention instrument and a collected instrument. They were tentatively developed by focus group among health providers and people with uncontrolled HT which was the prior step of this research project (Oba \& Chutipanyaporn, 2018).

First, the intervention instrument was the care process of the NTHMP or the program, including:

\section{Team-approached health education}

It was designed at the time of OPD visit divided into four sessions (30-45 min/session) for group health education. A physician taught on DASH dietary practices, a physical therapist worked on increasing individualized physical activity, a pharmacist taught how to take medication correctly and regularly, and a Thai traditional practitioner demonstrated stress reduction practices. After each session, there were open discussions on the practice which took around 15-20 minutes/session.

\section{Medication taking support}

The pharmacist checked the remaining medication of participants at each visit. If the pharmacist found out that some participants took medicine irregularly, then she wrote a small note in the patient's record for NP and the physician reviews and educated them on how to take the antihypertensive drug correctly.

\section{Home blood pressure monitoring (HBPM)}

The nurse practitioner demonstrated how participants can measure their own BP and suggested the time measured should be based on two measurements taken in the morning or two measurements taken at night over a preferred period of seven days. After learning from the NP, the participant practiced to measure their blood pressure in the correct position, how to read the results, and how to record the outcome by writing in a notebook.

\section{Motivation interviewing on behavioral adjustment}

The nurse practitioner applied the four steps of motivational interviewing (MI); engaging, focusing, evoking, and establishing, which was developed by Miller and Rollnick for helping participants explore and resolve their ambivalence problem as well as promoting behavioral adjustment (McNeil, Addicks, \& Randall, 2017).

Second, the collecting instrument was a form for recording the participant's office SBP and DBP at before, first month, second month, after the programmed interventions, and three months postintervention as well as the number of hypertensive urgencies experienced by each patient. This parameter was collected from their medical records.

The intervention instrument and the collecting data were evaluated for content validity by three independent experts: a medical doctor, a lecturer, and professional nurse. A consensus form of agreement was developed with a scale ranging from $+1=$ not relevant, $+2=$ item need some revision, $+3=$ relevant but need minor revision, $+4=$ very relevant, and included an opened-ended option for expert suggestions. This consensus form was based on the content validity index (CVI) technique. The values of the CVI calculations for two research instruments were 1.00 .

Researchers coordinated with the hospital director for asking permission to conduct the research. After permission was given, simple random sampling was used to find the participants as explained in detail in the samples section earlier. After respondents' agreement via mobile phone, researchers made an appointment with them for clarifying the objectives, intervention, and signed their ethic consent at the chronic care clinic before staring the program. There were 30 people with uncontrolled HT participated in this research. The researchers ran the NTHMP and collected the data at the chronic disease clinic as follow:

At the first day of the program, after 30 participants' baseline blood pressure levels (SBP and DBP) were collected by automatic blood pressure machine of chronic care clinic, two sessions of group health education, antihypertensive drug and selfmonitoring blood pressure demonstration were done in a hospital meeting room. The health educators were a pharmacist and researchers (NP) which took 20 and 30 minutes, respectively. These two activities were completed before participants met the physician for routine check-up and medication prescribing.

At first month visit, participants' blood pressure was collected by the chronic care clinic automatic blood pressure machine. Pharmacists checked each participant's remaining antihypertensive medications and asked about how to take each medication. Two sessions of group health education, DASH diet and benefits of physical activity and muscle-strengthening practice were done in a hospital meeting room. The health educators were a physician and physical therapist which took 30 and 20 minutes, respectively. Then, one by one, participants met the principle researcher who acted as NP the counseling room of the chronic disease clinic. NP review participants' BP home records and applied individual face to face brief MI of around 5-15 minutes/participant before participants met the physician.

At second month visit, the participants' blood pressure was collected by the chronic care clinic automatic blood pressure machine. The pharmacists 
checked each participant's remaining antihypertensive medications. One session of group health education, neck massage and foot soaking by Thai traditional practitioner was done for 30 minutes in a hospital meeting room. Then, the NP reviewed participants' BP records for identifying their performances. NP performed brief MI with participants one by one before participants met the physician.

At third month visit or after intervention, participants' blood pressure was collected by the chronic care clinic automatic blood pressure machine. Pharmacists checked each participant's remaining antihypertensive medications and asked about how to take each medication. NP reviewed participants' BP records for identifying their behavioral change. NP evaluated the participant behavioral adjustments and motivated them to continue their appropriate behaviors. Therefore, the intervention was delivered for three months. There were no respondent drop out during the intervention.

At three months post-intervention (three months after completing the intervention), thirty participants' blood pressures were collected by the chronic care clinic automatic blood pressure machine.

The SBP and DBP data were analyzed by using mean and standard deviation. The difference of the average mean of SBP and DBP was analyzed using repeated measured ANOVA. The number of hypertensive urgencies and the number of admissions reported for the sample were counted and percentages were analyzed.

Ethics clearance for this research was obtained from the Ethics Committee for Research and Human Studies of Naresuan University in Thailand (number
COA No.240/2014, October 3, 2017). The researchers provided details of the study to the participants prior to obtaining informed consent. Confidentiality was assured by code numbering in all data and only the lead investigator was able to identify names with individual participant responses. The researchers obtained written consent and participants understood they could withdraw from the study at any time without penalty to assure protection of participant rights.

\section{RESULTS}

Table 1 shows personal data of 30 participants: mostly were male $(63.3 \%)$ and female $(36.7 \%)$. Their ages were between $20-29$ years (3.3\%), $40-49$ years (6.7\%), 50-59 years (46.7\%), and $>60$ years $(43.3 \%)$. They finished primary school level (80.0\%), secondary school level and technical school (10.0\%), and bachelor's degree level (10.0\%). Their occupations were farmer $(56.7 \%)$, merchant (3.3\%), general contractor $(23.33 \%)$, office worker $(13.3 \%)$ and unemployed (3.3\%). They were diagnosed with HT for $1-5$ years $(76.7 \%), 6-10$ years $(20.0 \%)$ and $>10$ years (3.3\%).

Table 2 displays the blood pressure before, first month, second month, after intervention, and three months post- intervention of people with uncontrolled HT. The results showed both SBP and DBP were significantly lower than at before intervention levels $(\mathrm{p}<0.001)$.

Table 3 shows post intervention, blood pressure level ranges demonstrating greater the number of participants with levels of higher BP decreased. At three months post- intervention both SBP and DBP are mostly still in the same range with at post

Table 1. Demographic Characteristic of Participants in Nurse-led Team-Based Hypertension Management Program (NTHMP)

\begin{tabular}{lcc}
\multicolumn{1}{c}{ Demographic characteristic } & Number & Percent \\
\hline Sex & 19 & 63.3 \\
Male & 11 & 36.7 \\
Female & & 3.3 \\
Age (year) & 1 & - \\
20-29 & - & 6.7 \\
$30-39$ & 3 & 46.7 \\
$40-49$ & 14 & 43.3 \\
$50-59$ & 13 & 80.0 \\
$>60$ & & 10.0 \\
Education & 24 & 10.0 \\
Primary school & 3 & 56.7 \\
Secondary school /Technical school & 3 & 23.4 \\
Bachelor's degree & & 13.3 \\
Occupations & 17 & 3.3 \\
Farmer & 7 & 3.3 \\
General contractor & 4 & 76.7 \\
Office worker & 1 & 20.0 \\
Merchant & 1 & 3.3 \\
Unemployed & & \\
Duration of HT & 23 & 6 \\
1-5 years & 1 & \\
6-10 years & 1 & \\
$>$ 10 years & 1 & \\
\hline
\end{tabular}


Table 2. Systolic Blood Pressure and Diastolic Blood Pressure of People with Uncontrolled HT Before, First Month, Second Month, After Intervention, and Three Months Post-Intervention (N=30)

\begin{tabular}{|c|c|c|c|c|c|c|}
\hline Blood pressure (mmHg) & Mean & S.D. & Type III SS & df & $\mathbf{F}$ & p-value \\
\hline \multicolumn{7}{|l|}{ Systolic BP } \\
\hline Before intervention & 144.55 & 10.34 & & & & \\
\hline 1st month & 131.23 & 12.86 & 2818.26 & 1 & 25.46 & $0.000 * * *$ \\
\hline 2nd month & 130.18 & 12.94 & & & & \\
\hline after intervention & 125.48 & 12.89 & & & & \\
\hline 3 months post-intervention & 132.10 & 11.28 & & & & \\
\hline \multicolumn{7}{|l|}{ Diastolic BP } \\
\hline Before intervention & 90.38 & 5.06 & & & & \\
\hline 1st month & 78.85 & 7.98 & 2160.08 & 1 & 51.52 & $0.000 * * *$ \\
\hline 2nd month & 78.55 & 11.18 & & & & \\
\hline After intervention & 74.38 & 8.29 & & & & \\
\hline 3 months post-intervention & 79.03 & 7.63 & & & & \\
\hline
\end{tabular}

$* * * \mathrm{p}<0.001$

Table 3. Amount and Percentage of Blood Pressure among People with Uncontrolled HT Before, After Intervention, And Three Months Post- Intervention

\begin{tabular}{|c|c|c|c|c|c|c|}
\hline \multirow{2}{*}{$\begin{array}{c}\text { BP Ranges } \\
(\mathrm{mmHg})\end{array}$} & \multicolumn{2}{|c|}{ Before intervention } & \multicolumn{2}{|c|}{ After intervention } & \multicolumn{2}{|c|}{ Three months post- intervention } \\
\hline & $\mathrm{n}$ & $\%$ & $\mathrm{n}$ & $\%$ & $\mathrm{n}$ & $\%$ \\
\hline \multicolumn{7}{|l|}{ Systolic } \\
\hline $101-120$ & & & 12 & 40.0 & 8 & 26.7 \\
\hline $121-140$ & 13 & 43.3 & 16 & 53.3 & 17 & 56.7 \\
\hline $141-160$ & 15 & 50.0 & 1 & 3.3 & 5 & 16.6 \\
\hline $161-180$ & 2 & 6.7 & 1 & 3.3 & & \\
\hline Total & 30 & 100 & $30 *$ & 100 & 30 & 100 \\
\hline \multicolumn{7}{|l|}{ Diastolic } \\
\hline $61-70$ & & & 8 & 26.7 & 2 & 6.7 \\
\hline $71-80$ & & & 16 & 53.3 & 16 & 53.3 \\
\hline $81-90$ & 21 & 70.0 & 4 & 13.3 & 9 & 30.0 \\
\hline $91-100$ & 9 & 30.0 & 2 & 6.7 & 3 & 10.0 \\
\hline Total & 30 & 100 & $30 *$ & 100 & $30 *$ & 100 \\
\hline
\end{tabular}

*) no admission in hospital with signs and symptoms of hypertension urgency

intervention. In addition, no participants were admitted into the hospital with signs and symptoms of hypertensive urgency until at three months postintervention.

\section{DISCUSSION}

The results indicated that people with uncontrolled HT had lower systolic blood pressures and diastolic blood pressure compared with baseline levels ( $\mathrm{p}<0.001)$, and no hospital admissions. It indicates that NTHMP could be able to lower risk of cardiovascular disease, chronic kidney disease, and stroke as well as improving quality of hypertensive care. The four reasons for this effectiveness of program are as follows:

First, the nurse practitioner, a leader of NTHMP, designed the group health education by inviting the healthcare team of a community hospital to provide knowledge and skills into four topics, DASH diet, increasing physical activity, stress relaxation, and medication taking. These four topics were selected by evidence based which could be effective to lower blood pressure (Dechkong, 2017; Ozpulat et al. 2017; Silveira et al., 2019). DASH diet, a health education, was taught by a physician because there was no dietician position in small community hospitals in Thailand. In the belief that a physician is knowledgeable in the treatment of diseases, participants obeyed and followed the advice of the physician, which allowed them to adjust their eating behaviors until their blood pressure levels were reduced. Previous reviews found the modified DASH diet (Guo et al., 2021), progressive muscle relaxation technique (Kep, 2018), and the mindfulness-based stress reduction program (MBSR) (Lee et al., 2020) can markedly decreased systolic and diastolic blood pressure of hypertension patients. In addition, the intervention group who received a supportive educational program by an interdisciplinary health are team had significantly lower SBP and DBP than those of the control group (Rerkluenrit, Shi, \& Pramuansup, 2018). Therefore, group health education of NTHPM helped participants to have knowledge on diet consumption, adequate physical activities and stress relaxation technique which they could apply in their daily life and might result in blood pressure reduction.

Second, most of the participants in this research were low economic status. The nurse practitioner taught how participants could measure their own blood pressure and how to record and interpret the outcome. This research provided blood pressure machines for each participant to measure their blood pressure at home during intervention. Home blood 
pressure records helped participants identify their current blood pressure and remind to adjust their diet and physical activities day to day. Participants' blood pressure records were reviewed by NP at first, second and third month visit for clarifying daily BP at home. The participants who measured more blood pressure learned more on how to control behavioral change related to their blood pressure. There is supporting evidence that BP measurement at home helps remind patients to continuously take antihypertensive and leads to better control of BP (Thai Hypertension Society, 2019). Short-term home blood pressure monitoring significantly reduces office blood pressure and improves medication adherence (Muhammad et al., 2019). SMBP and cointerventions lead to clinically significant BP reduction (Tucker, et al, 2017). So, HMBP helps participants to keep their blood pressure at target level and to prevent dangerous high blood pressure level.

Third, from systematic review and meta-analysis of non-adherence of antihypertensive drug, it was found that non-adherence to antihypertensive medications was noticed in $45 \%$ of the subjects studied and a higher proportion of uncontrolled BP (83.7\%) (Melaku et al., 2017). This research planned to solve non-adherence of antihypertensive drug by cooperating with a pharmacist who is a member of the health team, to monitor antihypertensive taking of participants at their second-third visits. These activities helped participants take antihypertensive drug correctly and effectively and to ensure that all participants had received the full range of antihypertensive drug which could be effective to decrease blood pressure. Prior research found educational intervention alone may not improve patients' BP control and medication adherence to antihypertensive medications. Pharmacist-led interventions improved BP control and medication adherence through education, counseling, or a combination of both (Reeves et al., 2020). The medication taking monitoring was done by a pharmacist to lower blood pressure of participants through education and counseling.

Fourth, from literature review, it was found that $\mathrm{MI}$ is powerful for behavioral change among persons with hypertension (Dechkong, 2017; McNeil, Addicks, \& Randall, 2017; Silveira et al., 2019). In this research, NP applied face to face brief MI around 5-15 minutes/participant to ensure that all participants understood the necessity of behavioral change. MI did not waste the participant's time because it was performed while the patient was waiting to see a doctor. Similarly, a research found that motivational interviews can be used in the acquisition of a healthy nutrition habit by hypertensive individuals, the increase in their exercise levels, and regulation of blood pressure (Ozpulat, et al, 2017). From metaanalysis reviews, interventions based on MI only or those with coaches were the most effective in hypertension improvement and MI (face to face delivered) was more effective than phone delivered
(Zomahoun et al., 2017). MI by NP helped participants to confirm that they could have diet consumption, physical activities, and perform stress relaxation suitable for their lifestyle, which could lead to blood pressure reduction.

HBPM was proven to have high potential to improve BP control but its devices are more expensive for low-income clients which is not affordable as well as a high gain of hospital support. This technique could be recommended to be applied in cases of high-income people with HT. Nevertheless, the interested people may use selfblood pressure devices in sub-district health promotion hospitals (SHPH) nearby their homes.

MI on behavioral adjustment is a good technique which helps participants by thinking and planning for their lifestyle changes. Nurses in OPDs and NPs in the primary healthcare system could learn the application of MI or be trained this technique via technology communication (IT) or extraordinary learning resources and find out the proper time to play this role.

It is a good opportunity of NP in SHPH, which had no pharmacist working in order to work on medication taking support, especially in older adults and those with low health literacy.

NTHMP was applied in a small community hospital, which had lack of a dietitian who is the expert on diet consumption. NP and physician take more time to work on diet lifestyle changes of the participants.

\section{CONCLUSION}

The overall results showed that NTHMP was effective in lowering SBP and DBP and prevented hypertensive urgency episodes among people with uncontrolled HT. NTHMP is effective due to the design of the program, including team-approached health education, medication taking support, HBPM and MI on behavioral adjustment. The key successes of the program were participation among health providers and people with uncontrolled HT.

To strengthen the program testing approach, a two-group pre-posttest experimental design should be applied. Nurses in community hospitals/primary healthcare level could apply a nurse-led team-based hypertension management for people with uncontrolled HT by increasing other outcomes such as self-management skill, body mass index, and stress scale to clarify the approaches' effects on lifestyle modification. To extend the role of NPs on hypertensive care, a study on people with nonadjusted antihypertensive drug to continue their lifestyle practices and prevent the complications should be done.

\section{ACKNOWLEDGEMENT}

This research was funded by Fiscal year 2018 grants, Naresuan University, Mueng District. Phitsanulok Province, Thailand (R2560B158). Our special thanks go to all of the participants who participated in this 
study and Mr. Kenje Baris Gunda BSN, RN, Naresuan University, for his assistance in editing this article.

\section{REFERENCES}

Alsaigh SAS, Alanazi, M.D., Alkahtani, M.A., Alsinani, T.S.A., Abdullah, A.A., Alghamdi, T.A.,

Alabdrabalrasol, E.A., \& Alzahrani, T.A. (2017). Lifestyle modifications for hypertension management. The Egyptian Journal of Hospital Medicine, 70(12), 2152-2156. https://doi.org/10.12816/0045044.

Basile, J., \& Bloch, M. (2019). Overview of hypertension in adults. UpToDate.

Bersaoui, M., Baldew, S.M., Cornelis. N., Toelsie, J., \& Cornelissen. V.A. (2020). The effect of exercise training on blood pressure in African and Asian populations: A systematic review and metaanalysis of randomized controlled trials. Europian Journal of Preventive Cardiology. 27(5), 457-472. https://doi.org/10.1177/2047487319871233.

Dechkong, T. (Ed.). Motivational interviewing for NCDs; MI NCDs. Bangkok: Beyond Publishing; 2017.

Flack, J.M. \& Adekola, B. (2019). Blood pressure and the new ACC/AHA hypertension guidelines. Trends in Cardiovascular Medicine, 30,160-164. https://doi.org/10.1016/j.tcm.2019.05.003

Guo, R., Li, N., Yang, R, Liao, X-Y., Zhang, Y., Zhu, B-F., Zhao, Q.,...Lei, Y. (2021). Effects of the Modified DASH Diet on adults with elevated blood pressure or hypertension: A systematic review and metaanalysis. Front. Nutr. 8:725020. doi: $0.3389 /$ fnut.2021.725020

Health Data Center. (2020).The prevalence rate of well controlled patients with hypertension. Available from https:/hdcservice.moph.go.th/hdc/reports/repor t kpi.php? [in Thai].

Ho, J, K., Carnagarin, R., Matthews, V.B., \& Schlaich, M.P. (2019). Self-monitoring of blood pressure to guide titration of antihypertensive medication a new era in hypertension management? Cardiovascular Diagnosis and Therapy, 9(1), 94-99. https://doi.org/10.21037/cdt.2018.08.01

Kep, D.A.S. (2018). The effect of progressive muscle relaxation techniques to decrease blood pressure for patients with hypertension in Mataram. Prim Health Care, 8(4) DOI: 10.4172/21671079.1000309

Lee, E.K.P., Yeung, N.C.y., Xu, Z., Zhang, D., Yu, C.P. Pong, C. \& Wong, S.Y.S. (2020). Effect and acceptability of mindfulness-based stress reduction program on patients with elevated blood pressure or hypertension: A meta-analysis of randomized controlled trials. Hypertension, 76:1992-2001,

https://doi.org/10.1161/HYPERTENSIONAHA.12 0.16160
Mandini, S., Conconi, F., Mori E, Myers, J., Grazzi, G, \& Mazzoni, G. (2018). Walking and hypertension: Greater reductions in subjects with higher baseline systolic blood pressure following six months of guided walking. PeerJ, e5471. https://doi.org/10.7717/peerj.5471

McNeil, D.W., Addicks, S.H. \& Randall, C.L. (2017). Motivational interviewing and motivational interactions for health behavior change and maintenance. Oxford handbook online. Scholar Research

Review, https://doi.org/10.1093/oxfordhb/978019993529 1.013.21

Melaku, A.T., Abdulla, S., Alemayehu, G.E., Srikanth, B.A., Ahmed, E.A. (2017). Nonadherence to antihypertensive drugs: A systematic review and meta-analysis. Medicine, 96(4) (e5641). https://doi.org/10.1097/MD.0000000000005641

Muhammad, J., Jamial, M.M., \& Ishak, A. (2019). Home blood pressure monitoring has similar effects on office blood pressure and medication compliance as usual care. Korean Journal of Family Medicine, 40, 335-34. https://doi.org/10.4082/kjfm.18.0026.

Oba, N. \& Chutipanyaporn, N. (2018). Development of care service model for uncontrolled hypertensive patients. Journal of Nursing and Health Science, 12(1), 24-34.

Ozpulat, F. \& Emiroglu, O.N. (2017). The effect of the motivational interview method on the lifestyle of hypertensive individuals. New Trends and Issues Proceedings on Advances in Pure and Applied Sciences, 8, 76-82. https://www.propaas.eu

Patel, P., Ordunez, P., DiPette, D., Escobar, M.C., Hassell, T., Wyss, F., Hennis, A., Asma, S., ...Angell, S. (2016). Improved blood pressure control to reduce cardiovascular disease morbidity and mortality: The standardized hypertension treatment and prevention project. The Journal of Clinical Hypertension 18(2) https://doi.org/10.1111/jch.12861

Ponte Márquez, P.H., Feliu-Soler, A., Solé-Villa, M.J., Matas-Pericas, L., Filella-Agullo. D., RuizHerrerias, M., Soler-Ribaudi, J., ....Arroyo-Díaz, J.A. (2019). Benefits of mindfulness meditation in reducing blood pressure and stress in patients with arterial hypertension. Journal of Human

Hypertension, 33, 237-247. https:/www.nature.com/articles/s41371-0180130-6 Proia, K.K., Thota, A.B., Njie, G.J., Finnie, R.K.C., Hopkins, D.P., Mukhtar, Q., Pronk, N.P., Cooksey, T. (2014). Team-based care and improved blood pressure control: A community guidesystematic review. American Journal of Preventive Medicine, 47(1), 86-89. https://doi.org/10.1016/j.amepre.2014.03.004.

Reeves, L., Robinson, K., McClelland, T., Adedoyin, C.A., Broeseker, A., \& Adunlin, G. (2020). Interventions in the management of blood pressure control and adherence to antihypertensive medications: A systematic review of randomized controlled trials. Journal of 
Pharmacy

Practice, https://doi.org/10.1177/0897190020903573

Rerkluenrit, J., Shi, L., \& Pramuansup, R. (2018). The effects of a supportive educational program by an interdisciplinary health care team on health promotion behavior to heart failure prevention among patients with coronary artery disease and coexisting hypertension. Journal of Nursing and Health Care, 34(3), 40-47.

Rollnick, S. \& Allison, J. (2004). Motivational interviewing. In $\mathrm{N}$ Heather, $\mathrm{S}$ Sciences, $\mathrm{T}$. Stockwell (Eds.) The essential handbook of treatment and prevention of alcohol problems (pp. 105-116).

West Sussex: John Wiley \& Sons Ltd. Seangpraw, K., Auttama, N., Tonchoy, P., \& Panta, P. (2019). The effect of the behavior modification program dietary approaches to stop hypertension (DASH) on reducing the risk of hypertension among elderly patients in the rural community of Phayao, Thailand. Journal of Multidisciplinary Healthcare, 12,109-118 https://doi.org/10.2147/JMDH.S185569

Silveira, L.C.J., Aliti, G.B., Silva, E.M.D., Pimentel, R.P., Gus, M., \& Rabelo-Silva, E.R. (2019). Effect of motivational interviewing in hypertensive patients (MIdNIgHT): study protocol for a randomized controlled trial. Trials, 20,414. https://doi.org/10.1186/s13063-019-3486-1

Thai Hypertension Society. (2019). 2019 Thai Guidelines on the treatment of hypertension. Chiang Mai: Trick Think Publishing; 2019.

Thongdang, K., \& Promsiripaiboon, Y. (2015). Effect of group process to change health behavior of patients with hypertension at Watpradoo Health Promotion Hospital, Muang District, Surat Thani Province. Community Health Development Quarterly Khon Khen University, 3(2), 261-271. https://home.kku.ac.th/chd/index.php?option=co mattachments\&task=download\&id=118\&lang=th Tucker, K.L., Sheppard, J.P., Stevens, R., Bosworth, H.B., Bove, A.B., Bray, E.P., Earle, K., George, J.,Godwin, M., Green, B.B......McManus, R.J. (2017). Self-monitoring of blood pressure in hypertension: A systematic review and individual patient data meta-analysis. PLoS Medicine, 14(9) e1002389.

https://doi.org/10.1371/journal.pmed.1002389. 